\title{
Multiple Peripheral Neuropathies Adjacent to Necrotizing Myositis Related to Rhabdomyolysis
}

\author{
In Soo Kim, Woo Hyung Lee, Jae-Young Lim \\ Department of Rehabilitation Medicine, Seoul National University Bundang Hospital, Seoul National University College of Medicine, \\ Seongnam, Korea
}

Corresponding Author: Jae Young Lim, MD, $\mathrm{PhD}$ Department of Rehabilitation Medicine, Seoul National University College of Medicine, Seoul National University Bundang Hospital, 82 Gumi-ro 173, Bundang-gu, Seongnam 13620, Korea

Tel: $+82-31-787-7739$

Fax: +82-31-787-4056

E-mail:drlim1@snu.ac.kr

Received: March 4, 2017

Revised: March 5, 2017

Accepted: March 23, 2017

\begin{abstract}
Herein, we report an elderly man with lower limb weakness due to multiple peripheral neuropathies associated with rhabdomyolysis that was identified by electro-diagnosis and hip magnetic resonance imaging (MRI). He was diagnosed with acute kidney injury due to rhabdomyolysis following prolonged immobilization after alcoholic intoxication. He complained of thigh pain, hypoesthesia, and weakness in both lower extremities. The electro-diagnostic study showed near complete axonal loss of the sciatic nerve and partial axonal loss of the femoral and obturator nerve, between the gluteal region and the proximal thigh level, which was more severe on the right side than on the left. Hip MRI revealed extensive necrotizing myositis on the gluteal, psoas, and adductor muscles, and an inflammatory lesion around multiple peripheral nerves at the pelvic and proximal thigh level. The clinical course and prognosis were discussed as an example of a neurological complication arising from an inflammatory condition related to rhabdomyolysis.
\end{abstract}

Key Words: Neuropathy, Necrotizing myositis, Rhabdomyolysis

\section{INTRODUCTION}

Rhabdomyolysis is a syndrome of skeletal muscle breakdown with leakage of muscle contents, sometimes referred to as "dissolution of striped skeletal muscle"1). Acute muscle necrosis with swollen, tender muscles results in myoglobinuria, 'tea-colored' urine, and subsequent limb weakness. This results from the rapid breakdown of skeletal muscle fibers, which leads to the leakage of potentially toxic cellular contents into the systemic circulation. Serum creatine kinase (sCK) level is typically elevated by at least 10 times the upper limit of the normal range followed by a rapid reduction of sCK level to near normal values. Crush or compressive injuries and prolonged immobility may occur along with the development of diffuse muscle weakness. In a complicated case, rhabdomyolysis is combined with peripheral nerve injury, which is usually the main reason for delaying functional recovery. Swelling of the damaged muscle occasionally leads to compartment syndrome ${ }^{2,3}$. Local compression of the nerve within the compartment has been suggested as being the main mechanism underlying peripheral nerve injury related to rhabdomyolysis, but some cases have shown a wide range of peripheral nerve damage that cannot be explained by this mechanism ${ }^{4,5}$. In addition, neurological complications rarely occur in young adults, so aging or frailty may also be a significant factor ${ }^{4,6)}$. We encountered a patient with extensive involvement of the bilateral lower-limb peripheral nerves associated with pelvic muscle inflammation following rhabdomyolysis. We present this case report as an example of a neurological complication resulting from inflammation related to rhabdomyolysis.

\section{CASE REPORT}

A 76-year-old man fell in a drunken state 2 days prior to visiting the Emergency Department due to gradual progressive weakness and altered mentality. Second-degree $(20 \mathrm{~cm}$ $\times 10 \mathrm{~cm}$ ) burn lesions were detected on the posterior thigh, which may have occurred due to a prolonged period of lying or sitting on a hot floor. He was a chronic alcoholic, drinking 1-2 bottles of Soju each day, in addition to taking antidepressant medicines owing to a depressive mood and sleep disturbance. In the blood tests performed at the time of visiting the Emergency Department, several abnormal laboratory findings were observed including detected elevated blood urea nitrogen $47 \mathrm{mg} / \mathrm{dL}$ (normal range, $10-26 \mathrm{mg} / \mathrm{dL}$ ), creatinine $4.98 \mathrm{mg} / \mathrm{dL}$ (normal range, $0.6-1.2 \mathrm{mg} / \mathrm{dL}$ ) and cre- 
atinine kinase $>28,000 \mathrm{IU} / \mathrm{L}$ (normal range, 21-232 IU/L). He was diagnosed with acute kidney injury and uremic encephalopathy due to rhabdomyolysis and was admitted to the Internal Medicine Department. Supportive care including urine alkalinization and empirical antibiotics was maintained. For the management of his burn injury on both posterior thighs and alcohol dependence with depression, we consulted the Plastic Surgery and Psychiatry Departments.

The patient complained of weakness and hypesthesia in the bilateral lower extremities, which led him to be transferred to a rehabilitation department 10 days after admission. Neurological examination showed muscle strength of hip flexion with an Medical Research Council (MRC) grade of 2-/2- on the right/left side, respectively; knee extension with an MRC grade of 2/2; and knee flexion, ankle dorsiflexion, and ankle plantar flexion with an MRC grade of 1/1. Positive findings were observed in the Thomas test and Ely test in both legs, and hip flexor and extensor tightness were found. A nerve conduction study performed 20 days after the onset of lower extremity weakness revealed that the left sural nerve showed a reduced amplitude of sensory nerve action potential; moreover, the right sural, right lateral femoral cutaneous, and both superficial peroneal nerves showed no response. Electrodiagnostic examination revealed bilateral sciatic neuropathy with severe axonal loss, along with femoral and obturator neuropathy with moderate axonal loss around and below the gluteal level (Tables 1, 2). In pelvic MRI, we observed myonecrosis involving the bilateral pelvic and thigh muscles in both the gluteus medius and minimus near the iliac spine, right medial portion of the gluteus maximus, both obturator externus, rectus femoris, adductor brevis and iliopsoas muscles, which were associated with multiple reactive lymph node enlargements. In addition, perineural sheath enhancements of the bilateral sciatic, femoral and obturator nerves were found in close proximity to multiple necrotizing myositis lesions from the pelvis to the proximal thigh (Fig. 1). At discharge, the patient could walk (endurance, $30 \mathrm{~m}$ ) with the bilateral use of an ankle foot orthosis. At the time of hospital discharge, lower extremity muscle strength improved: hip flexion (MRC grade 4/4), hip extension (MRC grade 4/4), knee extension (MRC grade 3/3), knee flexion (MRC grade $3 / 3$ ), ankle dorsiflexion (MRC grade 1/2), and ankle plantar flexion (MRC grade 1/2). Six months after discharge, there were no specific changes in follow-up electro-diagnostic examinations. During the follow-up period, the patient still had difficulty in walking and right hip pain increased gradually. At the 2-year follow-up, the bilateral neuropathies remained in a stationary state with incomplete recovery, and newly detected avascular necrosis of the bilateral hip joints made his physical function worse.

\section{DISCUSSION}

Here, we report a case of rhabdomyolysis in an elderly patient that caused extensive pelvic muscle inflammation and involved the adjacent peripheral nerves. This case may have a clinical implication as an example of a neurological complication caused by inflammatory neuromyositis related

Table 1. Nerve conduction study results for the initial and follow-up electro-diagnostic examination

\begin{tabular}{|c|c|c|c|c|c|c|c|c|}
\hline \multirow[b]{2}{*}{ Motor \& sensory } & \multicolumn{4}{|c|}{ Initial } & \multicolumn{4}{|c|}{ Follow-up (6 months after discharge) } \\
\hline & $\begin{array}{c}\text { Latency } \\
(\mathrm{ms})\end{array}$ & $\begin{array}{c}\text { Amplitude } \\
(\mathrm{mV})\end{array}$ & $\begin{array}{c}\text { Velocity } \\
\text { (m/sec) }\end{array}$ & Response & $\begin{array}{c}\text { Latency } \\
(\mathrm{ms})\end{array}$ & $\begin{array}{c}\text { Amplitude } \\
(\mathrm{mV})\end{array}$ & $\begin{array}{l}\text { Velocity } \\
(\mathrm{m} / \mathrm{sec})\end{array}$ & Response \\
\hline \multicolumn{9}{|l|}{ Motor } \\
\hline Rt common peroneal - TA & & & & No & & & & No \\
\hline Lt common peroneal - TA & & & - & No & & & & No \\
\hline Rt common peroneal - EDB & & & - & No & & & & No \\
\hline Lt common peroneal - EDB & & & & No & 3.33 & 0.4 & 43.8 & \\
\hline Rt tibial - AH & & & & No & & & & No \\
\hline Lt tibial - AH & & & & No & & & & No \\
\hline Rt femoral - VM & & & & No & & & & \\
\hline Lt femoral - VM & 8.65 & 0.7 & & & & & & \\
\hline \multicolumn{9}{|l|}{ Sensory } \\
\hline Rt sural & & & & No & & & & No \\
\hline Lt sural & & & & No & & & & No \\
\hline $\mathrm{R}$ sup peroneal & & & & No & & & & No \\
\hline Lt sup peroneal & & & & No & & & & No \\
\hline Rt saphenous & & & & No & & & & No \\
\hline Lt saphenous & & & & No & & & & No \\
\hline
\end{tabular}

Rt, right; Lt. left; TA, tibialis anterior; EDB, extensor digitorum brevis; AH, abductor hallucis; VM, vastus medialis. 
Table 2. Electromyographic results for the initial and follow-up electro-diagnostic examination

\begin{tabular}{|c|c|c|c|c|c|c|c|c|}
\hline \multirow{2}{*}{ Muscle } & \multicolumn{4}{|c|}{ Initial } & \multicolumn{4}{|c|}{ Follow-up (6 months after discharge) } \\
\hline & Fib & PSW & Poly & Recruitment pattern & Fib & PSW & Poly & Recruitment pattern \\
\hline Rt vastus medialis & $1+$ & $1+$ & Increased & Reduced & $3+$ & $3+$ & Increased & Reduced \\
\hline Rt tibialis anterior & $2+$ & $2+$ & - & No activity & $2+$ & $2+$ & - & No activity \\
\hline Rt GCM & $2+$ & $2+$ & - & No activity & $3+$ & $3+$ & - & No activity \\
\hline Lt vastus medialis & $1+$ & $1+$ & $\mathrm{N}$ & Reduced & None & None & Increased & Reduced \\
\hline Lt tibialis anterior & $3+$ & $3+$ & - & No activity & $3+$ & $3+$ & Increased & Discrete \\
\hline Lt GCM & $3+$ & $3+$ & - & No activity & $3+$ & $3+$ & Increased & Single \\
\hline Rt adductor longus & $2+$ & $2+$ & - & No activity & $2+$ & $2+$ & Increased & Discrete \\
\hline Lt adductor longus & $3+$ & $3+$ & $\mathrm{N}$ & Single & $2+$ & $2+$ & Increased & Reduced \\
\hline Rt biceps femoris & $2+$ & $2+$ & - & No activity & $2+$ & $2+$ & Increased & Single \\
\hline Lt biceps femoris & $2+$ & $2+$ & Increased & Single & $2+$ & $2+$ & Increased & Discrete \\
\hline Rt gluteal maximus & $2+$ & $2+$ & $\mathrm{N}$ & $\mathrm{N}$ & None & None & $\mathrm{N}$ & $\mathrm{N}$ \\
\hline Lt gluteal maximus & None & None & $\mathrm{N}$ & $\mathrm{N}$ & None & None & $\mathrm{N}$ & $\mathrm{N}$ \\
\hline Rt lumbar paraspinal & None & None & $\mathrm{N}$ & $\mathrm{N}$ & None & None & $\mathrm{N}$ & $\mathrm{N}$ \\
\hline
\end{tabular}

Fib, fibrillation potential; PSW, positive sharp wave; Poly, polyphasic motor unit action potential; Rt, right; Lt. left; GCM, gastrocnemius; $\mathrm{N}$, normal.

to rhabdomyolysis. As a case that supports an inflammatory cause, our current patient could be included in the current debate surrounding the mechanism of rhabdomyolysis-related neuropathy.

The development of rhabdomyolysis is intricately intertwined with a diverse spectrum of factors affecting muscle membranes, ion channels, and muscle energy supply. Despite various causes, an uncontrolled elevation in free intracellular calcium and activation of calcium-dependent proteases, which lead to the destruction of muscle fiber contents, have been established as common pathophysiological mechanisms ${ }^{1)}$.

The elderly have been reported to be more susceptible to the development of rhabdomyolysis and more likely to have complications related to rhabdomyolysis ${ }^{6-8)}$. Rhabdomyolysis is commonly diagnosed in intoxicated patients subjected to prolonged muscle compression as they lay motionless, and in elderly patients following a fall or stroke ${ }^{9)}$. Two mechanisms can thus be considered for the development of rhabdomyolysis following drug or alcohol intoxication, or prolonged immobilization. One is primarily the toxic effects of the drug directly upon the muscle cells while the other relates to the increased need for oxygen due to immobility, seizures, trauma, and metabolic abnormalities ${ }^{10)}$. In the present case, prolonged lying or sitting on a hot floor with low consciousness, due to heavy alcoholic ingestion, is likely to be a leading factor in the development of rhabdomyolysis.

Several etiologies have been suggested to explain the mechanism of neurological complications such as peripheral nerve damage following rhabdomyolysis. Compartment syndrome is not a rare finding in rhabdomyolysis, and leads to the formation of local edema and an increase in intramus- cular pressure which can induce compressive neuropathy ${ }^{3)}$.

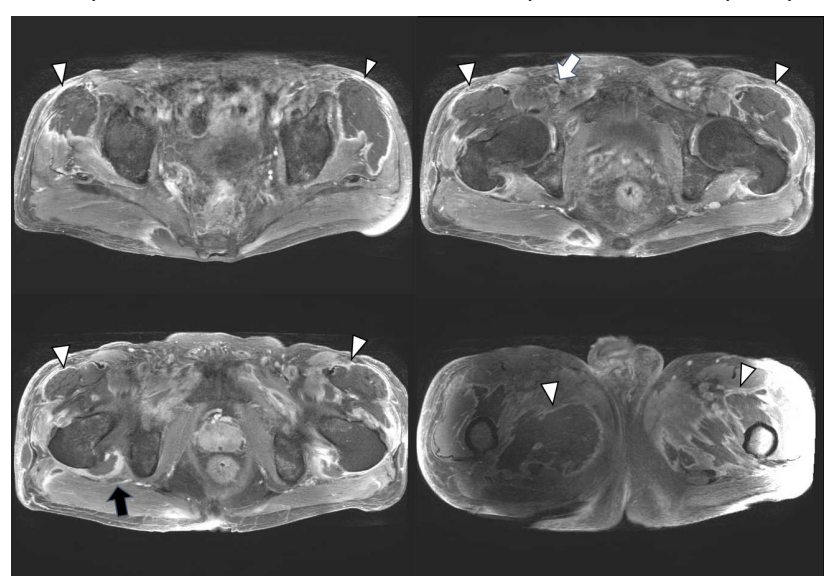

Fig. 1. Pelvic magnetic resonance imaging (MRI) showed myonecrosis (arrowhead) involving the bilateral pelvic and thigh muscles associated with multiple reactive lymph node enlargements. Perineural sheath enhancements of the right sciatic nerve (black arrow) and the femoral nerve (white arrow) were found in close proximity to multiple necrotizing myositis lesions from the pelvis to the proximal thigh.

However, compartment syndrome alone could not explain the mechanisms underlying severe bilateral neuropathies in the lower extremities. Several reports support the fact that muscle damage can induce local neuroinflammation and ischemia $^{11,12)}$. Lee and Lim $^{4)}$ have suggested that nerve damage occurs due to the spread of inflammation associated with rhabdomyolysis. In this previous case, the nerve injury site was located in the proximal portion of the biceps femoris. In other words, the main lesion was located outside of the buttock compartment, not within the buttock compartment. 
Therefore, regardless of the pressure increase in the buttock compartment, it could be judged that the inflammation spread caused severe nerve damage. In our case, MRI revealed inflammatory change on the sciatic, femoral and obturator nerves associated with the extensive involvement of inflammatory myonecrosis in the bilateral pelvic and proximal thigh areas. Our case is similar to a previous case reported by Lee and $\mathrm{Lim}^{4)}$ in that it is a case of rhabdomyolysis not confined to a compartment or compression mechanism. However, our case was more extensive with more profound damage than previous cases. The possibilities of neural inflammation related to necrotic muscles may be supported by the following manifestations in our current case: (1) Whole peripheral nerves of the lower extremity including the femoral, obturator and sciatic nerves were involved. (2) The range of nerve injury was quite extensive, including bilateral involvement and with a relatively long segmental lesion in each individual nerve. (3) T2-weighted MRI showed high signal intensity around the perineural tissues adjacent to the necrotic pelvic and thigh muscles.

Until now, we have known very little about the prognosis of a patient with neurological complications related to rhabdomyolysis. Electro-diagnostic findings in our current case, performed during the initial phase and during follow-up, suggested a poor prognosis for neurological recovery. He cannot walk independently due to weakness and poor balance, even after more than 2 years of follow-up. We found that neurological complications related to rhabdomyolysis showed poor prognosis in this particular case. Therefore, early detection and appropriate management should be warranted to minimize morbidities from these complications, especially in older patients.

Conflicts of Interest Disclosures: The researchers claim no conflicts of interest.

\section{REFERENCES}

1. Warren JD, Blumbergs PC, Thompson PD. Rhabdomyolysis: a review. Muscle Nerve 2002;25:332-47.

2. Alobaidi A, Backdash MM, El-Menyar A. Thigh compartment syndrome complicated by sciatic nerve palsy, rhabdomyolysis, and acute renal failure. Clin Case Rep 2015;4:107-10.

3. Chung KJ, Chung YK, Yoo JH, Wang JS. Sciatic nerve palsy complicating gluteal compartment syndrome due to rhabdomyolysis: a case report. J Kor Orthop Assoc 2005;40:103-6.

4. Lee SA, Lim JY. Bilateral sciatic neuropathy associated with rhabdomyolysis in an immobilized patient: a case report. J Korean Acad Rehabil Med 2009;33:127-30.

5. Roth JV. Bilateral sciatic and femoral neuropathies, rhabdomyolysis, and acute renal failure caused by positioning during radical retropubic prostatectomy. Anesth Analg 2007;105: 1747-8.

6. Ginanneschi F, Volpi N, Giannini F, Rocchi R, Donati D, Aglianò $\mathrm{M}$, et al. Rhabdomyolysis in an elderly multitreated patient: multiple drug interactions after statin withdrawal. J Neurol Sci 2014;336:284-7.

7. Bhardwaj S, Selvarajah S, Schneider EB. Muscular effects of statins in the elderly female: a review. Clin Interv Aging 2013;8: 47-59.

8. Gareri P, Segura-García C, De Fazio P, De Fazio S, De Sarro G. Sertraline-induced rhabdomyolysis in an elderly patient with dementia and comorbidities. Ann Pharmacother 2009;43:1354-9.

9. Huerta-Alardín AL, Varon J, Marik PE. Bench-to-bedside review: Rhabdomyolysis: an overview for clinicians. Crit Care 2005;9:158-69.

10. Prendergast BD, George CF. Drug-induced rhabdomyolysis: mechanisms and management. Postgrad Med J 1993;69:333-6.

11. Hamel Y, Mamoune A, Mauvais FX, Habarou F, Lallement L, Romero NB, et al. Acute rhabdomyolysis and inflammation. J Inherit Metab Dis 2015;38:621-8.

12. Dabby R, Djaldetti R, Gilad R, Herman O, Frand J, Sadeh M, et al. Acute heroin-related neuropathy. J Peripher Nerv Syst 2006;11:304-9. 